\title{
FORUM
}

\section{Autonomie und Steuerung verselbstständigter Behörden: Management, Regulierung und die Bedeutung formaler Strukturen}

\author{
Tobias Bach
}

\begin{abstract}
Autonomy and Control of Agencies: Management, Regulation and the Importance of Formal Structures
\end{abstract}

Abstract: In an article published in PVS Ebinger and Schmitt (2010) investigate the effects of structural devolution to federal agencies on administrative performance. They address a topic which has not gained much attention in German-language public sector research. The authors perform a comprehensive and sound statistical analysis of unique survey data. However, their literature review, case selection, and theoretical model are seriously flawed. The present article critically addresses these problems and develops suggestions for further research.

Keywords: Agencies, Delegation, Performance, Federal Administration, Germany

Schlagwörter: Agencies, Delegation, Performanz, Bundesverwaltung, Deutschland

\section{Einleitung ${ }^{1}$}

In einem in der PVS veröffentlichten Aufsatz (Ebinger u. Schmitt 2010) wird die seit einigen Jahren lebhaft geführte Diskussion über Autonomie und Steuerung verselbstständigter Behörden aufgegriffen (Bach et al. 2010; Bouckaert u. Peters 2004; Christensen u. Lægreid 2006; Döhler 2005, 2007a, 2007b; Gilardi u. Braun 2002; Greve et al. 1999; Pollitt et al. 2001, 2004; Thatcher 2002; Verhoest et al. 2004, 2010; Wettenhall 2005). Als zentrale Forschungslücke identifizieren Ebinger und Schmitt (S. 70) den fehlenden empirischen Nachweis eines kausalen Zusammenhangs zwischen organisatorischer Verselbstständigung sowie effizienter und effektiver Aufgabenwahrnehmung. ${ }^{2}$ Das sogenannte Praktiker-Modell organisatorischer Verselbstständigung geht davon aus, dass die Delegation von Entscheidungskompetenzen im Bereich des internen Managements (z. B. durch Globalbudgets) zu einer effizienteren und effektiveren Verwaltung führt (Pollitt et al. 2004, S. 30-43). Effizienz und Effektivität der öffentlichen Verwaltung sind je-

1 Ich möchte mich bei Marian Döhler, Markus Seyfried, den anonymen Gutachtern und der PVSRedaktion für konstruktive Hinweise zu früheren Fassungen dieses Beitrags bedanken.

2 Zur Verbesserung der Lesbarkeit wird auf eine wiederholte Nennung des vollständigen Literaturhinweises verzichtet und ggf. auf die Seitenzahlen verwiesen. 
doch unklar definiert und - wenn überhaupt - nur unter erheblichem methodischem Aufwand messbar. Die Autoren argumentieren daher, dass ein erweiterter Entscheidungsspielraum der Führungskräfte verselbstständigter Behörden im Verhältnis zur politischen Führung eine notwendige Bedingung für erhöhte administrative Leistungsfähigkeit sei. Die Führungskräfte nutzen diesen Entscheidungsspielraum, indem sie ihre Entscheidungen stärker an fachlichen als an (partei) politischen Kriterien ausrichten, wodurch eine effiziente und effektive Aufgabenwahrnehmung begünstigt werde (S. 70, 75).

Dieses Argument untersuchen die Autoren anhand einer umfassenden Befragung administrativer Führungskräfte in Ministerialverwaltung und verselbstständigten Behörden auf Bundesebene. Sie kommen zu dem Ergebnis, dass die wahrgenommene Handlungsfreiheit der Führungskräfte mit der Häufigkeit hierarchischer Interventionen durch die politische Führung und der politischen Relevanz des Tätigkeitsbereichs abnimmt (S. 87-88). Zudem weisen sie nach, dass Führungskräfte in Ministerien stärker funktional politisiert sind als in verselbstständigten Behörden und dass Führungskräfte „unabhängiger Verwaltungseinheiten “ weniger stark politisiert sind als „die Leitung abhängiger Bundesoberbehörden“ (S. 87). Entgegen den theoretischen Erwartungen der Autoren haben die Entscheidungsspielräume bei der internen Behördensteuerung keinen Einfluss auf die funktionale Politisierung administrativer Führungskräfte. Hieraus ziehen sie den Schluss, dass „die häufig praktizierte Überlassung von Management-Autonomie nicht den erhofften Effekt zu haben scheint" (S. 88) und somit eine zentrale Annahme „der Agenturforschung“ (S. 87) widerlegt sei.

Ebinger und Schmitt widmen sich einem in der deutschsprachigen Verwaltungsforschung wenig beachteten Forschungsfeld und können auf einzigartige Daten zurückgreifen, die umfassend und nachvollziehbar statistisch analysiert werden. Demgegenüber weisen die Darstellung des Forschungsstandes, die Fallauswahl und die theoretische Argumentation erhebliche Schwächen auf, wodurch die Aussagekraft der empirischen Analyse und der hieraus gezogenen Schlussfolgerungen deutlich eingeschränkt wird. Ebinger und Schmitt verweisen mehrfach auf „die Agenturforschung“ (S. 71, 72, 75, 83, 87, 88). In der politik- und verwaltungswissenschaftlichen Literatur gibt es „die“ Agenturforschung jedoch nicht; vielmehr werden zwei sehr unterschiedliche Debatten zu verselbstständigten Behörden geführt, die im Hinblick auf die politische Steuerung der Behörden auf widersprüchlichen Annahmen beruhen. Zudem argumentieren die Autoren, dass formale Organisationsmerkmale verselbstständigter Behörden wie Rechtsform oder ministerielle Weisungsrechte für deren tatsächliche Autonomie keine Bedeutung haben (Fußnote 7, S. 76). Dieses Argument beruht offensichtlich auf einer Fehlinterpretation der einschlägigen Literatur (Döhler 2005, 2007a, 2007b). Schließlich führen ein deutlicher selection bias bei der Fallauswahl und eine nicht nachvollziehbare Unterscheidung abhängiger und unabhängiger Behörden zu erheblichen Problemen bei der Interpretation der empirischen Analyse. Der Beitrag endet damit, dass mögliche Anknüpfungspunkte an den Stand der Forschung aufgezeigt werden. 


\section{Begriffsbestimmung: Agenturen, Behörden, Quangos}

Die seit einigen Jahren intensiv geführte Debatte zu verselbstständigten Behörden nimmt ihren Ausgangspunkt in der empirischen Beobachtung, dass die Anzahl sogenannter agencies seit dem Ende der 1980er-Jahre in zahlreichen OECD-Ländern sprunghaft angestiegen ist bzw. dass neue Formen der leistungsorientierten Steuerung (z. B. Zielvereinbarungen, Kennzahlen) dort eingeführt wurden, wo solche Behörden bereits seit längerer Zeit vorhanden waren (Pollitt et al. 2001; Thatcher 2002). Allerdings bleibt in dieser Diskussion vielfach unklar, welche öffentlichen Organisationen als agencies bezeichnet werden können und worin sich diese - sofern es sich um einen eigenen Organisationstypus handelt - von anderen öffentlichen Organisationen unterscheiden (Greve et al. 1999; Pollitt et al. 2004; Smullen 2004; Thynne 2004; Wettenhall 2005).

\subsection{Was ist eine „Agentur“?}

Der Begriff agency wurde in der englischsprachigen Literatur schon lange vor dem "agency fever" (Pollitt et al. 2001) der vergangenen beiden Jahrzehnte verwendet und bedeutet zunächst nichts anderes als öffentliche Behörde oder Amt (Hood 1978; Thynne 2004). ${ }^{3}$ Ebinger und Schmitt argumentieren, dass agency ein „Oberbegriff für sehr unterschiedliche organisatorische Arrangements“ (S. 71) sei, der in der Literatur mit ähnlichen Konzepten wie non-departmental public bodies, hybrids, quangos, fringe bodies, oder quasi-autonomous public organizations beliebig austauschbar verwendet werde. ${ }^{4}$ In der Bundesverwaltung wird der Begriff „Agentur“ seit einigen Jahren für öffentlich und privatrechtlich verfasste Behörden und Einrichtungen der Bundesverwaltung verwendet (Döhler 2007b). ${ }^{5}$ Auch im englischsprachigen Raum kann von einer einheitlichen Verwendung des Begriffs keine Rede sein (Thynne 2004). Für die politisch-administrative Praxis trifft die Einschätzung der Autoren, dass es sich um einen eher hemdsärmelig verwendeten Oberbegriff handelt, offenbar zu.

In der akademischen Diskussion hingegen wird der Agenturbegriff vor allem dafür kritisiert, dass er viel zu eng gefasst sei, wodurch zahlreiche andere Organisationstypen künstlich ausgeklammert werden (Wettenhall 2005, S. 620; siehe auch Christensen u. Lægreid 2006, S. 12). Die Wahrnehmung öffentlicher Aufgaben durch Organisationen außerhalb der Ministerialverwaltung sei weder ein neues Phänomen, noch gebe es hierzu keine Forschung, was in der aktuellen Literatur immer wieder beklagt werde (Wettenhall 2005, S. 620). Es gibt jedoch plau-

3 Gilardi und Braun (2002) verwenden als deutsche Übersetzung den Begriff „Ämter“.

4 In diesem Zusammenhang zitieren die Autoren (S. 71) wörtlich aus Greve et al. (1999). Der zitierte Satz ist dort allerdings nicht auffindbar.

5 Die Bundesagentur für Arbeit trägt diese Bezeichnung seit 2004 und ist eine Körperschaft des öffentlichen Rechts. Die Bundesnetzagentur hat ihren heutigen Namen 2005 erhalten; sie ist eine Bundesoberbehörde. Die Deutsche Arzneimittel- und Medizinprodukteagentur, deren Errichtung 2007 scheiterte, war als Anstalt des öffentlichen Rechts geplant. Die Bundesagentur für Außenwirtschaft, welche 2009 in der Germany Trade and Invest - Gesellschaft für Außenwirtschaft und Standortmarketing mbH aufging, wurde seit 2001 als solche bezeichnet. Die Bundesrepublik Deutschland Finanzagentur GmbH wurde 2000 gegründet. 
sible Argumente für eine analytische Eingrenzung des Begriffs, was insbesondere für international vergleichende Forschungsdesigns Voraussetzung ist (Bouckaert u. Peters 2004; Greve et al. 1999, S. 139; Pollitt et al. 2004; Verhoest et al. 2010).

In der neueren Literatur wird überwiegend die Definition von Pollitt et al. (2001, S. 274-275, 2004, S. 10; siehe auch Thynne 2004, S. 96; Verhoest et al. 2010, S. 17-18) verwendet, die auch Ebinger und Schmitt (S. 71-72) ihrer Argumentation zugrunde legen. Die Wahrnehmung öffentlicher Aufgaben durch Agenturen bzw. verselbstständigte Behörden ist demnach eine vergleichsweise schwache Form der organisatorischen Verselbstständigung, die von anderen Formen der Verselbstständigung abzugrenzen ist, welche auf eine noch größere Distanz zur Ministerialverwaltung abzielen (Greve et al. 1999, S. 142; Pollitt et al. 2001, S. 275-276). Im Vergleich zu vollständig oder teilweise privatisierten öffentlichen Unternehmen befinden sich Agenturen bspw. durch ministerielle Weisungsbefugnisse und finanzielle Abhängigkeiten immer noch im unmittelbaren Einflussbereich der Ministerialverwaltung, worin sich auch das politikwissenschaftliche Forschungsinteresse an verselbstständigten Behörden begründet.

Die Frage, welche Merkmale konstitutiv für eine Agentur sind, kann nur in Abgrenzung zu anderen Formen der organisatorischen Verselbstständigung sinnvoll beantwortet werden (siehe für Deutschland Bach u. Jann 2010, S. 449-450). In den vergangenen Jahren wurden zahlreiche Typologien organisatorischer Verselbstständigung vorgelegt (Bouckaert u. Peters 2004; Greve et al. 1999; Pollitt et al. 2004; Thynne 2004), die von Ebinger und Schmitt zwar mehrheitlich wahrgenommen werden, deren zentrales Argument eines „Kontinuums“ öffentlicher Leistungserstellung aber unberücksichtigt bleibt. Letztlich muss sich eine Begriffsbestimmung, sofern es sich bei agencies und agencification nicht lediglich um Modebegriffe aus der Praxis handelt, auch gegenüber anderen Begriffsbestimmungen bewähren und eigene Forschungsfragen aufwerfen (Thynne 2004, S. 96-98). Dass dies in der Literatur aus sehr unterschiedlichen Perspektiven geschieht, wird im folgenden Abschnitt näher beleuchtet.

\subsection{Agenturen in der empirischen Verwaltungsforschung: Management und Regulierung}

Ebinger und Schmitt berufen sich an mehreren Stellen ihres Beitrags auf „die Agenturforschung “ (S. 71, 72, 75, 83, 87, 88) und streben eine empirische Überprüfung der „postulierten Ziele“ (S. 75) und der „theoretischen Prämissen der Agenturbildung“ (S. 75) an. Mein zentrales Argument lautet, dass es „die“ Agenturforschung nicht gibt. Vielmehr muss zwischen einer managementorientierten und einer auf unabhängige Regulierungsbehörden fokussierten Debatte unterschieden werden, die insbesondere im Hinblick auf das Ausmaß der politischen Steuerung der Behörden auf widersprüchlichen Annahmen beruhen (Bach u. Jann 2010, S. 445; Bach et al. 2010, S. 15-22; Christensen u. Lægreid 2006, S. 8; Döhler 2007b, S. 12-13). Im Zusammenhang mit den Motiven für Delegation berufen sich die Autoren in erheblichem Umfang auf die Literatur zu unabhängigen Regulierungsbehörden; ihr zentrales theoretisches Argument zu den erwarteten Effek- 
ten organisatorischer Verselbstständigung beruht jedoch ausschließlich auf der managementorientierten Debatte.

In der durch das New Public Management (NPM) geprägten managementorientierten Literatur stehen von Ebinger und Schmitt hervorgehobene Aspekte wie Effizienz und Effektivität der öffentlichen Verwaltung im Mittelpunkt (S. 70, 75, 87). Ihren Ausgangspunkt nimmt diese Debatte in den sogenannten Next-StepsReformen in Großbritannien Ende der 1980er-Jahre (Bach et al. 2010; Bouckaert u. Peters 2004). Die bereits angesprochene enge Definition des Begriffs „Agentur“ wird seitdem mit einer Reihe spezifischer Ideen verknüpft, die zu einem Idealtypus moderner Agenturen verdichtet wurden (Pollitt et al. 2004, S. 30-46; Smullen 2004, S. 184-185). Eine moderne Agentur ist demnach eine verselbstständigte Behörde, die nicht über hierarchische Steuerungsinstrumente (z. B. Weisungen) und detaillierte Budget- und Personalvorgaben, sondern über Ziel- und Leistungsvereinbarungen gesteuert wird und über erweiterte Handlungsspielräume für das interne Management (z. B. Personal, Finanzen, Organisation) verfügt. Diese Merkmale (strukturelle Verselbstständigung, Kontraktsteuerung, ManagementAutonomie) werden in der Literatur auch als Dreibeiner-Modell bezeichnet (Bach et al. 2010, S. 16-17; Pollitt et al. 2004, S. 30-46).

Als weiteres Merkmal einer modernen Agentur wird die Trennung von Politikformulierung (Ministerialverwaltung) und -umsetzung (Agentur) genannt (Verhoest et al. 2010, S. 8). Diese Trennung wurde beispielsweise in Flandern (Verschuere 2009, S. 27-28) oder Neuseeland (Christensen u. Lægreid 2001, S. 80) als explizites Ziel managementorientierter Agenturreformen verfolgt. Pollitt et al. (2004, S. 4142) hingegen argumentieren, dass zahlreiche empirische Beispiele für Politikvorbereitung und -formulierung durch verselbstständigte Behörden vorliegen, weshalb die Trennung von policy und operations kein zwingendes Merkmal der idealtypischen modernen Agentur sondern „a sub-variant within the agency trend" sei (Pollitt et al. 2004, S. 42). Es handelt sich jedoch zweifelsohne um eine besonders wichtige Dimension des Verhältnisses von Ministerialverwaltung und verselbstständigten Behörden, die beim Vergleich zwischen der idealtypischen modernen Agentur und der unabhängigen Regulierungsbehörde berücksichtigt werden sollte (Tabelle 1).

Tabelle 1: Idealtypen moderner Agenturen und unabhängiger Regulierungsbehörden im Vergleich

\begin{tabular}{|c|c|c|}
\hline & moderne Agentur & unabhängige Regulierungsbehörde \\
\hline Struktur & - strukturell verselbstständigt & - strukturell verselbstständigt \\
\hline Steuerung & $\begin{array}{l}\text { - Ziel- und } \\
\text { Leistungsvereinbarungen }\end{array}$ & $\begin{array}{l}\text { - hierarchische Steuerung } \\
\text { (gesetzlich) eingeschränkt }\end{array}$ \\
\hline Autonomie & $\begin{array}{l}\text { - Management-Autonomie } \\
\text { (Finanzen, Personal, interne } \\
\text { Organisation) }\end{array}$ & $\begin{array}{l}\text { - inhaltliche Autonomie bei } \\
\text { regulatorischen Entscheidungen }\end{array}$ \\
\hline $\begin{array}{l}\text { Politikformulierung } \\
\text { und -entscheidung }\end{array}$ & $\begin{array}{l}\text { - Ministerium bzw. Parlament } \\
\text { - ggf. Einbindung der Behörde } \\
\text { durch Ministerium }\end{array}$ & $\begin{array}{l}\text { - Ministerium bzw. Parlament } \\
\text { (Rahmengesetzgebung) } \\
\text { - Behörde (Regulierungsstandards) }\end{array}$ \\
\hline Implementation & - Behörde & - Behörde \\
\hline
\end{tabular}

Quelle: eigene Darstellung. 
Die managementorientierte Literatur bezieht sich zumeist auf die sogenannte Praktiker-Theorie organisatorischer Verselbstständigung, wonach die (gleichzeitige) Umsetzung der idealtypischen Merkmale einer modernen Agentur zu Effizienz- und Effektivitätsgewinnen führt (Pollitt et al. 2004, S. 30-43; Verhoest et al. 2010, S. 6-7). In Anlehnung an diese Perspektive argumentieren Ebinger und Schmitt, dass eine operative Führung verselbstständigter Behörden ,jenseits politischer Prärogativen“ (S. 75) die entscheidende Voraussetzung für effizientes und effektives Verwaltungshandeln sei. In der Literatur wird jedoch genau umgekehrt die Stärkung der politischen Steuerung verselbstständigter Behörden durch Zielund Leistungsvereinbarungen als zentrales Ziel managementorientierter Verwaltungsreformen beschrieben (Christensen u. Lægreid 2001, S. 78; Döhler 2005, S. 220). Die Verselbstständigung von Behörden nach dem NPM-Modell müsste demnach zu einer stärkeren Berücksichtigung politischer Vorgaben durch die Behörden bei einer gleichzeitigen Erweiterung operativer Handlungsspielräume führen. Daher kann dem Argument der Autoren nicht zugestimmt werden, dass die Verselbstständigung von Behörden nach dem Vorbild des NPM darauf abzielt, die funktionale Politisierung der Behördenleitung zu minimieren (S. 75).

Weitestgehend getrennt von der managementorientierten Debatte wird seit einigen Jahren eine politik- und verwaltungswissenschaftliche Debatte zu unabhängigen Regulierungsbehörden geführt, die auch als independent regulatory agencies oder non-majoritarian institutions bezeichnet werden (Döhler 2006; Christensen u. Lægreid 2006; Elgie 2006; Gilardi 2002; Gilardi u. Braun 2002; Majone 1997; Thatcher 2002). Auch diesem Behördentypus wird ein sprunghaftes Wachstum in den vergangenen Jahren attestiert, das als Begleiterscheinung der Liberalisierung und Privatisierung ehemaliger staatlicher Monopole und als institutionelle Antwort auf die zunehmende Komplexität politischer Probleme gesehen wird (Majone 1997; als Zusammenfassung der US-Literatur siehe Pollack 2002). Die Delegation weitreichender inhaltlicher Entscheidungsbefugnisse an unabhängige Regulierungsbehörden wird insbesondere mit dem funktionalistischen Argument der glaubhaften Verpflichtung (credible commitment) politischer Entscheidungsträger begründet. Für letztere besteht demnach ein großer Anreiz, zur Erreichung kurzfristiger politischer Ziele von bereits getroffenen Entscheidungen abzuweichen, die langfristig zu effizienteren Ergebnissen führen würden. Indem politische Entscheidungsträger weitreichende Entscheidungskompetenzen an Regulierungsbehörden delegieren, die vor direkten politischen Eingriffen geschützt („unabhängig“) sind, erhöhen sie ihre Glaubwürdigkeit gegenüber den Adressaten (Elgie 2006, S. 208; Gilardi 2002, S. 874-876; Majone 1997, S. 153-154; Thatcher 2002, S. 129-131). Die formale Einschränkung direkter politischer Eingriffsmöglichkeiten stellt demnach ein zentrales Merkmal der idealtypischen unabhängigen Regulierungsbehörde dar.

Im Vergleich zum Idealtypus der modernen Agentur werden die Merkmale unabhängiger Regulierungsbehörden besonders deutlich (Tabelle 1). Die entscheidenden Unterschiede bestehen zum einen in der größeren Entscheidungsteilhabe in der Politikformulierung bzw. den Ermessensspielräumen unabhängiger Regulierungsbehörden bei inhaltlichen Entscheidungen, und zum anderen in der „fachlich bedingten Autonomisierung “ (Döhler 2006, S. 223) regulativer Behörden, die 
mit begrenzten Möglichkeiten der direkten politischen Steuerung einhergeht (siehe auch Christensen u. Lægreid 2006, S. 23). ${ }^{6}$ Während eine idealtypische moderne Agentur einer vergleichsweise starken politischen Steuerung durch Leistungsvorgaben unterliegt, kann hiervon bei unabhängigen Regulierungsbehörden gerade nicht ausgegangen werden. Insofern sind die theoretischen Annahmen von Ebinger und Schmitt zum Zusammenhang von Verselbstständigung und administrativem Handlungsspielraum für (unabhängige) Regulierungsbehörden durchaus nachvollziehbar; diese werden aber aus der managementorientierten Literatur abgeleitet, die diesbezüglich zu völlig anderen Schlussfolgerungen kommt.

\section{3. ,Agenturfieber' in Deutschland?}

Ebinger und Schmitt deuten mehrfach an, dass auch in Deutschland empirische Hinweise auf ein „Agenturfieber“ vorliegen. Den Abschluss von Ziel- und Leistungsvereinbarungen beschreiben sie als zentrales Merkmal moderner Agenturen (S. 72). Zudem verweisen die Autoren auf die „lange Geschichte der verselbstständigten Verwaltungseinheiten " (S. 72) in Deutschland, machen aber auch deutlich, dass bestimmte Veränderungen zu beobachten sind, die als Anzeichen einer Zunahme von Agenturen bzw. als vorsichtige Annäherung an das moderne Agenturmodell gedeutet werden (Fußnote 9, S. 78, S. 70). Hierzu zählen die Autoren die Gründung neuer Behörden ,insbesondere mit Aufsichts- und Regulierungsaufgaben [...], die verstärkte Einstellung von Quereinsteigern aus der Wirtschaft" sowie den „häufige[n] Abschluss von Zielvereinbarungen zwischen Ministerien und nachgeordneten Behörden “ (Fußnote 9, S. 78). Allerdings wird keine schlüssige Operationalisierung moderner Agenturen vorgenommen, was umso problematischer einzuschätzen ist, als die Abgrenzung zwischen „traditionellen Verwaltungseinheiten und Agenturen" (S. 88) in der Fallauswahl und der empirischen Analyse eine zentrale Rolle spielt (S. 79). Zunächst einmal sind jedoch die empirischen Beobachtungen der Autoren grundsätzlich infrage zu stellen.

Die Anzahl der unmittelbaren Bundesbehörden hat innerhalb der vergangenen zwei Jahrzehnte nicht zugenommen, sondern abgenommen (Bach u. Jann 2010, S. 447). Diese Entwicklung fand überwiegend als Zusammenschluss einzelner Bundesbehörden (z. B. Forschungsanstalten des BMELV) statt. Ebenso ist eine verstärkte Abwanderung von Behörden der unmittelbaren Verwaltung in die mittelbare (z. B. Bundesanstalt für Finanzdienstleistungsaufsicht, BaFin) oder die privatrechtlich organisierte Verwaltung (z. B. Deutsche Flugsicherung $\mathrm{GmbH}$ ) zu beobachten. Demgegenüber fand eine Übertragung größerer Aufgabenblöcke aus der Ministerialverwaltung an nachgeordnete Behörden nur in sehr begrenztem Umfang statt (z. B. ehemalige Regulierungsbehörde für Telekommunikation und Post, heute Bundesnetzagentur) (Bach u. Jann 2010, S. 456-458). Eine möglicherweise nachhaltig wirksame Veränderung der Topografie verselbstständigter Bundesbehörden ist eher auf der inhaltlichen Ebene der von den Behörden wahrgenommenen Aufgaben zu sehen, und zwar insbesondere im Bereich der Markt- und

6 Eine ausführlichere Diskussion der Merkmale regulativer Verwaltung findet sich bei Döhler (2006). 
Risikoregulierung (Döhler 2006, S. 210). Eine „Verselbstständigung von Verwaltungseinheiten“ (S. 77) durch die Gründung neuer Behörden hingegen ist im bundesdeutschen Kontext nur sehr eingeschränkt zu beobachten.

Im Hinblick auf die Anwendung von Zielvereinbarungen beziehen sich Ebinger und Schmitt (Fußnote 2, S. 72) auf die Zahlen der Bundesregierung aus dem Jahre 2005, wonach mit knapp der Hälfte der nachgeordneten Bundesbehörden (203 von 429) Zielvereinbarungen abgeschlossen wurden. ${ }^{7}$ Gemäß den offiziellen Zahlen hat sich die Anzahl verselbstständigter Bundesbehörden mit Zielvereinbarungen innerhalb weniger Jahre erheblich erhöht (Bach et al. 2010, S. 58). Eine 2008 durchgeführte Befragung verselbstständigter Behörden kommt hingegen zu dem Ergebnis, dass nur knapp 38 Prozent der Behörden eine Zielvereinbarung mit dem Ministerium abgeschlossen haben, und hiervon wiederum nur etwas mehr als die Hälfte messbare Vorgaben enthalten (Bach u. Jann 2010, S. 461). Während Ebinger und Schmitt (S. 78, Fußnote 9) von einem „häufige[n] Abschluss von Zielvereinbarungen zwischen Ministerien und nachgeordneten Behörden“ ausgehen, erscheint es angesichts dieser Ergebnisse eher zweifelhaft, ob Zielvereinbarungen klassisch-hierarchische Steuerungsmechanismen verdrängt haben (Bach u. Jann 2010, S. 462; Döhler 2007b, Fußnote 2, S. 13). Allein von der Anzahl der abgeschlossenen Zielvereinbarungen kann zudem kaum auf deren Relevanz für die Steuerung der Behörden geschlossen werden.

Schließlich gehen die Autoren davon aus, dass zunehmend Quereinsteiger aus der Privatwirtschaft in verselbstständigten Bundesbehörden tätig sind (Fußnote 9, S. 78), und zwar insbesondere bei Regulierungsbehörden (S. 82). In diesem Zusammenhang vermuten sie, dass „die externe Rekrutierung von Führungspersonal“ (S. 77-78) zu einer Veränderung von Verhaltensstandards geführt hat. Eine Untersuchung der Karrieremuster der Leiter der Bundesoberbehörden $(\mathrm{N}=41$, Stichjahr 2009) zeigt hingegen, dass lediglich fünf Prozent der Behördenleiter Seiteneinsteiger mit einer unmittelbaren Vorposition in der Privatwirtschaft sind (Hert 2009, S. 18-19). Beispielsweise war unter den Präsidenten der wichtigsten marktregulativen Behörden und deren direkten Vorgängern lediglich der Präsident der Bundesnetzagentur, Matthias Kurth, in der Privatwirtschaft tätig (allerdings weniger als ein Jahr). ${ }^{8}$ Ein weiteres prominentes Beispiel im Bereich der Leistungsverwaltung ist der Vorstandsvorsitzende der Bundesagentur für Arbeit, Frank-Jürgen Weise, der vor seiner derzeitigen Tätigkeit überwiegend in der Privatwirtschaft tätig war. Hierbei handelt es sich aber offensichtlich um Einzelfälle.

7 Laut der von den Autoren zitierten Antwort der Bundesregierung auf eine parlamentarische Anfrage (BT-Drs. 15/5111) bezieht sich die „Angabe [...] auf Zielvereinbarungen zwischen nachgeordneten Behörden und ihrer jeweils direkt vorgesetzten Behörde“ (S. 6). In die Gesamtzahl von 445 Behörden (einschließlich der Bundesministerien) gehen allerdings auch 27 Bundesmittelbehörden und 324 untere Bundesbehörden ein (S. 3). Letztlich bleibt also unklar, wie viele Zielvereinbarungen tatsächlich zwischen Ministerien und unmittelbar nachgeordneten Behörden abgeschlossen wurden.

$8 \mathrm{Zu}$ diesen Behörden zählen die Bundesnetzagentur, das Bundeskartellamt und die Bundesanstalt für Finanzdienstleistungsaufsicht (Döhler 2006, S. 212). Auch im Bundesinstitut für Arzneimittel und Medizinprodukte, das von den Autoren beispielhaft erwähnt wird (Fußnote 9, S. 78) verfügen weder der aktuelle Präsident noch dessen Vorgänger über Erfahrung in der Privatwirtschaft (Stand: Dezember 2010). 
Möglicherweise sind verselbstständigte Behörden unterhalb der obersten Führungsebene durchlässiger gegenüber der Privatwirtschaft geworden; an der Spitze dieser Behörden ist ein solcher Trend aber nicht erkennbar.

\section{Die Bedeutung der formalen Autonomie verselbstständigter Behörden während und nach der Delegation}

Ebinger und Schmitt (Fußnote 7, S. 76) schließen die Rechtsform verselbständigter Behörden explizit aus ihrem Erklärungsmodell aus. Die Autoren argumentieren unter Verweis auf Döhler (2007b, S. 27-28), dass „die Wahl der Rechtsform in der bundesdeutschen Verwaltung von einem hohen Maß an Willkür gekennzeichnet“ und die Rechtsform einer Behörde daher „mit keinen besonderen Autonomierechten verbunden“ sei. Daher könne die Rechtsform einer Behörde auch „keinen Beitrag zur Erklärung der abhängigen Variable leisten“ (Fußnote 7, S. 76).

Das zentrale Problem dieser Argumentation besteht darin, dass zwei unterschiedliche Phasen der Verwaltungssteuerung miteinander vermischt werden, nämlich die legislative Steuerung, welche die Rechtsformenwahl und die inhaltliche Programmierung verselbstständigter Behörden beinhaltet, und die prozedurale Steuerung, welche die ministerielle Steuerungspraxis umfasst (Döhler 2007a, S. 38-39, 320-322). Die fehlende Abgrenzung dieser beiden Phasen führt dazu, dass die Argumentation von Döhler (2005, 2007a, 2007b) hinsichtlich der zugrundeliegenden Motive und des Entscheidungsspielraums in der Phase der legislativen Programmierung in problematischer Weise auf die Phase der prozeduralen Steuerung übertragen wird. Der Ausgangspunkt für die Analyse dieser beiden Phasen sind US-amerikanische Theorien für die Erklärung von Delegationsentscheidungen.

\subsection{Institutional choice in parlamentarischen Regierungssystemen: Wieso rationalistische Theorien die Bedeutung der Rechtsform überschätzen}

In der einschlägigen US-Literatur wird das Verhältnis von Politik und Verwaltung üblicherweise mithilfe des Prinzipal-Agent-Modells analysiert, welches einen Prozess der Delegation von einem Auftraggeber (hier: Politik) an einen Auftragnehmer (hier: Verwaltung) beschreibt, die divergierende Präferenzen besitzen und danach streben, ihren individuellen Nutzen zu maximieren (Gilardi u. Braun 2002, S. 149-152). Das zentrale Problem für die politische Steuerung der Verwaltung besteht in der Informationsasymmetrie zugunsten des Auftragnehmers, welche letzterem starke Anreize bietet, von den Vorgaben des Auftraggebers abzuweichen (Döhler 2005, S. 216; Gilardi u. Braun, S. 149).

Zur Verwirklichung ihrer Präferenzen versuchen Politiker - so die US-Argumentation - schon in der Phase der legislativen Programmierung durch die Festlegung hierarchischer Weisungsrechte, parlamentarischer Anhörungsrechte oder begrenzter Ermessensspielräume ihre zukünftigen Einflusschancen zu sichern. Diese formalen Behördenmerkmale werden wiederum als Mechanismen zur Steuerung und Kontrolle der Verwaltung interpretiert, die Ergebnis eines rationalen, 
auf Nutzenmaximierung zielenden Entscheidungsprozesses sind. Die legislative Steuerung ist diejenige Phase „where principals make their strategic decisions about the powers delegated to agents, and the control mechanisms established to limit their autonomy" (Pollack 2002, S. 206). Unabhängigkeit und Entscheidungskompetenzen der Behörden gelten somit als „Resultat eines mit eindeutigen materiell-politischen Zielen versehenen Maximierungsverhaltens [...], das vor allem aus dem Misstrauen gegenüber fehlender Folgebereitschaft der Verwaltung geschürt wird“ (Döhler 2005, S. 222).

In der Literatur mehren sich die Stimmen, dass diese Ansätze für institutional choice in parlamentarischen Regierungssystemen eine deutlich eingeschränkte Erklärungskraft besitzen (Döhler 2005, 2007a, 2007b; Pollack 2002; Yesilkagit u. Christensen 2010). Wird dieses Modell auf den bundesdeutschen Kontext angewendet „besteht das zentrale Problem [...] darin, dass aus der Existenz von Strukturen oder Regelungen, die Steuerungswirkungen auf die Verwaltung haben können, auf politische Steuerungsintentionen geschlossen wird, die genau das zum Ziel haben" (Döhler 2005, S. 226). Tatsächlich werde bei Delegationsentscheidungen auf „erprobte Standardlösungen“ (Döhler 2005, S. 225) zurückgegriffen, wodurch die Unsicherheit politischer Entscheidungsträger über mögliche Folgewirkungen von Delegationsentscheidungen reduziert werde. Ähnlich argumentieren Yesilkagit und Christensen (2010), die anhand einer empirischen Analyse regulativer Behörden in Dänemark, den Niederlanden und Schweden zeigen, dass Institutional-Choice-Prozesse durch eingeübte Muster der Delegation bestimmt sind, während aus rationalistischen Modellen abgeleitete Erklärungen keinen Beitrag zur Erklärung der formalen Autonomie der untersuchten Behörden leisten. Auch Thatcher (2002) betont, dass funktionalistische Erklärungen für die Errichtung unabhängiger Regulierungsbehörden zu kurz greifen und in erheblichem Maße durch den jeweiligen politisch-administrativen Kontext bestimmt werden.

Es bleibt festzuhalten, dass in parlamentarischen Regierungssystemen ein absichtsvolles Design von Mechanismen zur politischen Kontrolle der Verwaltung nicht beobachtet werden kann, was in der US-amerikanischen Diskussion und in der Literatur zu unabhängigen Regulierungsbehörden in Europa aber vielfach unterstellt wird (Döhler 2005; Yesilkagit u. Christensen 2010). Dass die Rechtsform einer Behörde nicht als Instrument zur Durchsetzung politischer Interessen genutzt wird, bedeutet jedoch nicht, dass die Rechtsform keinerlei Bedeutung für die tatsächliche Autonomie verselbstständigter Behörden besitzt.

\subsection{Der Zusammenhang zwischen formaler und tatsächlicher Autonomie}

In den vergangenen Jahren kann zunehmend beobachtet werden, dass Behörden und Einrichtungen der unmittelbaren Bundesverwaltung in Einrichtungen der mittelbaren Bundesverwaltung (insbesondere Anstalten des öffentlichen Rechts) unter Beibehaltung der Fachaufsicht umgewandelt wurden oder eine solche Umwandlung geplant war (Bach u. Jann 2010; Döhler 2007b). Darüber hinaus werden sogar privatrechtliche Organisationen, die öffentliche Aufgaben wahrnehmen, der Fachaufsicht unterstellt (Döhler 2007b, S. 27-28). Dieser vergleichsweise gro- 
ße Entscheidungsspielraum bei der Festlegung der Rechtsform und der Ausgestaltung struktureller Merkmale verselbstständigter Behörden (Bach u. Jann 2010, S. 450; Döhler 2007b, S. 18-22) verleitet Ebinger und Schmitt (Fußnote 7, S. 76) zur Schlussfolgerung, dass die Rechtsformenwahl mehr oder weniger willkürlich stattfindet. Dabei übersehen die Autoren, dass mit der Wahl der Rechtsform von der mittelbaren Verwaltung über die unmittelbare Verwaltung, die Bundesprivatverwaltung und die Bundesverrichtungsverwaltung eine „graduelle Abnahme hierarchisch-bürokratischer Regelbindung “ (Döhler 2007b, S. 20) verbunden ist. Die Rechtsform hat demnach erheblichen Einfluss auf verschiedene Dimensionen formaler Autonomie verselbstständigter Behörden und zieht deutliche „Konsequenzen für das Steuerungsinstrumentarium “ (Döhler 2005, S. 222) nach sich. Während die Behörden und Einrichtungen der unmittelbaren Bundesverwaltung das öffentliche Personal- und Haushaltsrecht zwingend anwenden müssen, kann deren Anwendung in der mittelbaren Bundesverwaltung gesetzlich eingeschränkt oder aufgehoben werden. Tatsächlich dient die Umwandlung von Bundesoberbehörden in rechtsfähige Anstalten oder die Delegation öffentlicher Aufgaben an privatrechtliche Organisationen häufig gerade dazu, dem öffentlichen Dienst- und Haushaltsrecht zu entfliehen (Döhler 2007b, S. 20).

Im Hinblick auf die von den Autoren genannten Dimensionen behördlicher Autonomie (S. 74) ist davon auszugehen, dass Behörden mit eigener Rechtspersönlichkeit oder privatrechtlich verfasste Teile der Bundesverwaltung über eine größere Management-Autonomie als Behörden der unmittelbaren Bundesverwaltung (z. B. Bundesoberbehörden) verfügen. Die verselbstständigten Behörden der mittelbaren Verwaltung nehmen beispielsweise hinsichtlich finanzieller Entscheidungen eine größere Autonomie wahr als diejenigen der unmittelbaren Verwaltung (Bach 2010, S. 101-105). Ein vergleichbarer Zusammenhang zwischen der Rechtsform und unterschiedlichen Dimensionen der Management-Autonomie besteht auch in anderen kontinentaleuropäischen Ländern (Österreich, Schweiz, Niederlande) (Bach u. Huber 2012) und in Flandern (Verhoest et al. 2004). Eine begrenzte hierarchische Aufsicht (d. h. Rechtsaufsicht, aber keine Fachaufsicht) als weitere Dimension der formalen Autonomie hat zudem einen von der Rechtsform unabhängigen Einfluss auf die wahrgenommene Implementations-Autonomie, also auf die Handlungsspielräume bei der Wahrnehmung der inhaltlichen Aufgaben der Behörde (Bach 2010, S. 101-105). Hieraus kann geschlussfolgert werden, dass „hybride“ Behörden tatsächlich mehr Management-Autonomie als Behörden der unmittelbaren Verwaltung besitzen, sich aber hinsichtlich ihrer inhaltlichen Entscheidungsspielräume nicht von diesen unterscheiden. ${ }^{9}$

Der Einfluss der Rechtsform auf die tatsächliche Autonomie verselbstständigter Behörden konnte in zahlreichen empirischen Studien nachgewiesen werden, wobei sich dieser Zusammenhang auf unterschiedliche Dimensionen der Manage-

9 In der Begründung des Gesetzes zur Errichtung der BaFin heißt es hierzu: „Die Bundesanstalt ist [...] - ebenso wie in der Vergangenheit die drei Aufsichtsämter - der Rechts- und Fachaufsicht des Bundesministeriums der Finanzen unterstellt. Durch den Anstaltsstatus und die damit verbundene Lösung vom Bundeshaushalt gewinnt die Anstalt jedoch mehr Unabhängigkeit im budgetären, organisationsrechtlichen und personellen Bereich, was auch auf den operativen Bereich durchschlägt“ (BT-Drs. 14/7033, S. 33). 
ment-Autonomie (Finanzen, Personal) beschränkt. Aus der vergleichsweise großen Entscheidungsfreiheit bei der Rechtsformenwahl kann daher nicht automatisch darauf geschlossen werden, dass die Rechtsform „mit keinen besonderen Autonomierechten verbunden ist" (Fußnote 7, S. 76).

\section{Welcher Zusammenhang besteht zwischen funktionaler Politisierung und administrativer Leistungsfähigkeit?}

Die bisherigen Ausführungen hatten die theoretischen und konzeptionellen Annahmen des Beitrags von Ebinger und Schmitt zum Gegenstand. Im folgenden Abschnitt stehen methodische Aspekte und die empirische Analyse der Autoren im Vordergrund.

\subsection{Fallauswahl}

Ebinger und Schmitt (S. 71-72) beziehen sich explizit auf die vergleichsweise eng gefasste Definition verselbstständigter Behörden von Pollitt et al. (2001). Der Fallauswahl liegt jedoch ein deutlich breiteres Verständnis verselbstständigter Behörden zugrunde, welches auch privatrechtlich verfasste Einrichtungen mit öffentlichen Aufgaben einschließt (S. 79). Eine derart breite Fallauswahl ist nicht per se problematisch. Beispielsweise können die (privatrechtlich verfassten) Durchführungsorganisationen der Entwicklungszusammenarbeit durchaus als verselbstständigte Behörden im funktionalen Sinne eingeordnet werden (Bach u. Jann 2010, S. 450). Allerdings beruht das theoretische Argument der Autoren maßgeblich auf der Literatur zu verselbstständigten Behörden mit einer vergleichsweise geringen formalen Autonomie. Zudem ist davon auszugehen, dass sich privatrechtliche Einrichtungen hinsichtlich der Management-Autonomie erheblich von öffentlich-rechtlich verfassten Behörden unterscheiden. In der empirischen Analyse werden diese Unterschiede jedoch nicht berücksichtigt.

Das zentrale Problem ist die Selektivität bei der Auswahl der Grundgesamtheit. Die Autoren verweisen darauf, dass nur ,jene Bundesoberbehörden in die Analyse einbezogen [wurden], deren Aufgabenbereich zumindest potenziell einen politisch relevanten Entscheidungsspielraum aufweist“ (S. 79) und zusätzlich „auch solche Behörden der mittelbaren Bundesverwaltung und privatrechtlich organisierte Einheiten einbezogen [wurden], die trotz ihrer Rechtsform der Fachaufsicht eines Ministeriums unterliegen“ (Fußnote 11, S. 79). Welche Kriterien bei der Bestimmung des Merkmals der politischen Relevanz zugrunde gelegt wurden, legen die Autoren jedoch nicht offen. Somit bleibt auch unklar, auf welche Grundgesamtheit sich die empirischen Ergebnisse beziehen, denn im Anhang sind lediglich diejenigen Behörden verzeichnet, die sich an der Befragung beteiligt haben.

In diesem Zusammenhang wird deutlich, dass die Fallauswahl eine systematische Verzerrung der Ergebnisse erwarten lässt. Die funktionale Politisierung des administrativen Führungspersonals kann nicht als unabhängig von der politischen Relevanz einer Behörde angesehen werden. Eine Fallauswahl anhand extremer Ausprägungen der abhängigen Variablen führt dazu, dass die Stärke der kausalen Effekte der erklärenden Variablen systematisch unterschätzt wird (Collier u. Ma- 
honey 1996, S. 60-63). Der von den Autoren erwartete Effekt organisatorischer Verselbständigung, nämlich eine schwächer ausgeprägte Berücksichtigung (partei) politischer Entscheidungskriterien, müsste ja gerade bei weniger politisch relevanten Behörden nachweisbar sein. Indem solche vermeintlich unwichtigen Behörden bei der Fallauswahl nicht berücksichtigt werden, wird die Varianz der abhängigen Variable systematisch reduziert.

\subsection{Traditionelle Behörden und moderne Agenturen?}

Ebinger und Schmitt (S. 72) heben hervor, dass die Bundesverwaltung aufgrund „ihrer großen Spanne an Organisations- und Steuerungsformen, die eine Differenzierung in Subgruppen mit unterschiedlichen Autonomiegraden erlaubt" besonders gut zur Untersuchung des Zusammenhangs zwischen Verselbstständigung und Leistungsfähigkeit geeignet sei. In ihrer Analyse orientieren sich die Autoren allerdings nicht an objektivierbaren Kriterien wie Rechtsform oder hierarchischen Weisungsrechten, sondern unterscheiden zwischen „abhängigen Bundesoberbehörden “ und „unabhängigen Bundesoberbehörden“ (S. 79) bzw. „traditionellen Verwaltungseinheiten und Agenturen“ (S. 88) und erläutern diese Abgrenzung folgendermaßen:

Diese Aufteilung beruht auf Experteneinschätzungen und berücksichtigt den besonderen rechtlichen Status sowie informell zugestandene Freiheiten in Form von „Gewohnheitsrechten“ einzelner Verwaltungseinheiten wie bspw. hinsichtlich Weisungsfreiheit, Benehmensfreiheit in Kernfragen und öffentlichen Stellungnahmen, weitestgehend unparteiische Besetzung der Führungsposten etc. (Fußnote 14, S. 79-80).

Die unterschiedlichen „Organisations- und Steuerungsformen“ der Bundesverwaltung werden also gerade nicht zur „Differenzierung in Subgruppen“ verwendet (S. 72). Die zur Abgrenzung verwendeten Kriterien sind nicht intersubjektiv nachvollziehbar, werden aber im Anhang entsprechend dieser Einteilung zumindest aufgeführt. ${ }^{10}$ Eine Unterscheidung nach Rechtsformen hingegen könnte zur Überprüfung des Zusammenhangs zwischen formaler und wahrgenommener Autonomie verselbstständigter Behörden genutzt werden.

Zudem argumentieren Ebinger und Schmitt, dass verselbstständigte Behörden in der bundesdeutschen Verwaltung „üblicherweise“ der Fach- und Rechtsaufsicht unterliegen (Fußnote 7, S. 76). Daher sei hinsichtlich der formalen Möglichkeiten politischer Einflussnahme auf die Behörden „über die Verwaltungseinheiten hinweg keine Varianz" vorhanden (Fußnote 7, S. 76). Hiermit wird begründet, dass die in der einschlägigen Literatur als structural autonomy bezeichnete Dimension bürokratischer Autonomie bei der Analyse nicht berücksichtigt werden müsse (Christensen 2001; Verhoest et al. 2004; Yesilkagit u. Christensen 2010). Auch wenn eine uneingeschränkte Fachaufsicht den Regelfall darstellt (Döhler

10 Die Liste enthält mehrere offensichtliche Fehler: Die BRD Finanzagentur GmbH wird sowohl als „Bundesoberbehörde“ als auch als „unabhängige Bundesoberbehörde“ eingeordnet. Die als „unabhängige Bundesoberbehörde “ eingeordnete Bundeszentrale für politische Bildung wird zweimal genannt. 
2005), unterliegen nicht alle verselbstständigten Bundesbehörden einer uneingeschränkten Fach- und Rechtsaufsicht. Beispielsweise unterliegen einige Behörden nur teilweise der Fachaufsicht (z. B. Bundesagentur für Arbeit) oder sind hiervon gänzlich ausgenommen (z. B. Bundesprüfstelle für jugendgefährdende Medien) (weitere Beispiele für sog. „ministerialfreie Räume“ bei Döhler 2007a, S. 103). ${ }^{11}$

Zudem übersehen die Autoren, dass structural autonomy nicht durch eingeschränkte oder fehlende ministerielle Weisungsrechte, sondern durch das Vorhandensein eines Verwaltungsrates und den ministeriellen Einfluss auf dessen Zusammensetzung definiert wird (Christensen 2001, S. 121-122; Verhoest et al. 2004, S. 105-108; Yesilkagit u. Christensen 2010, S. 63). Wird die Geschäftsführung durch einen Verwaltungsrat überwacht, verringern sich die direkten Einflussmöglichkeiten der politischen Führung (Christensen 2001) und damit möglicherweise auch das Ausmaß funktionaler Politisierung der administrativen Führungskräfte. ${ }^{12}$

\subsection{Erklärungsmodell und Analyse}

Das Erklärungsmodell von Ebinger und Schmitt (S. 74-75) beruht auf der Annahme, dass der Grad der funktionalen Politisierung des administrativen Führungspersonals in einem negativen Zusammenhang mit dessen operativem Handlungsspielraum steht. Die Variable zur Messung der funktionalen Politisierung bildet ab, inwieweit die Handlungsorientierungen administrativer Führungskräfte durch (1) die politische Zustimmungsfähigkeit von Entscheidungsalternativen, (2) die Antizipation der Präferenzen der politischen Führung sowie (3) die Abhängigkeit des eigenen Verantwortungsbereichs von der politischen Führung geprägt sind ( $\mathrm{S}$. 80, 88). Die Autoren beziehen sich zudem auf eine Definition funktionaler Politisierung, welche die Berücksichtigung und Antizipation politischer Entscheidungskriterien („policy proposals and legislative drafts“) bei der Gesetzesvorbereitung umfasst (S. 75). Insofern bleibt unklar, welcher Zusammenhang zwischen funktionaler Politisierung und operativem Handlungsspielraum administrativer Führungskräfte besteht.

Die Autoren machen mehrfach deutlich, dass die Autonomie verselbstständigter Behörden und das Ausmaß der funktionalen Politisierung im Spannungsfeld zwischen dem „politischen Prinzipal“ und der verselbstständigten Behörde angesehen wird (S. 70, 74, 76, 77, 78, 88). Im Zusammenhang mit einer Analyse der Ministerialverwaltung lässt sich möglicherweise vom politischen Prinzipal sprechen; im Hinblick auf verselbstständigte Behörden erscheint dies jedoch äußerst problematisch. Ein Behördenleiter bekommt den zuständigen Minister vergleichsweise selten zu Gesicht; für die Autonomie verselbstständigter Behörden sind vielmehr die Steuerungsambitionen und -fähigkeiten der Ministerialverwaltung ausschlaggebend (Döhler 2007a). Insofern ist zu hinterfragen, ob die Häufigkeit

11 Auch die Stichprobe der Autoren enthält Behörden, die keiner (Bundesbeauftragte für die Unterlagen des Staatssicherheitsdienstes der ehemaligen DDR) oder einer eingeschränkten Fachaufsicht (Bundesinstitut für Risikobewertung) unterliegen.

12 Ein Verwaltungsrat ist in Deutschland insbesondere bei den Behörden der mittelbaren Bundesverwaltung üblich (z. B. Bundesagentur für Arbeit, Bundesanstalt für Finanzdienstleistungsaufsicht) (Bach et al. 2010, S. 40-41). 
formaler und informeller Weisungen der politischen Führung ein valides Konstrukt für die Messung der „Policy-Autonomie“ (S. 89) verselbstständigter Behörden ist. Die empirische Forschung zum Steuerungsverhalten der Ministerialverwaltung zeigt zudem, dass letztere vor allem im Hinblick auf administrative Aspekte steuernd eingreift, während eine inhaltliche, aufgabenbezogene Steuerung deutlich schwächer ausgeprägt ist (Bach 2010).

Die Autoren können nachweisen, dass funktionale Politisierung, die Häufigkeit inhaltlicher Weisungen durch die politische Führung und die wahrgenommene politische Bedeutung der eigenen Aufgabe in einem signifikanten Zusammenhang stehen (S. 86). Dieses Ergebnis deutet darauf hin, dass die funktionale Politisierung durch die jeweilige Verwaltungsaufgabe maßgeblich beeinflusst wird. Es ist hingegen wenig verwunderlich, dass zwischen der Management-Autonomie verselbstständigter Behörden und der funktionalen Politisierung des Führungspersonals kein Zusammenhang nachgewiesen werden kann, da hierdurch unterschiedliche Dimensionen organisatorischer Autonomie erfasst werden, die unabhängig voneinander variieren können (Bach 2010; Christensen 2001; Verhoest et al. 2004).

Die Autoren können zweifelsohne beanspruchen, eine relevante Dimension der Beziehungen zwischen Ministerialverwaltung und verselbstständigten Behörden zu erfassen. Hieraus lassen sich jedoch keine Rückschlüsse auf die operativen Handlungsspielräume der Behörden ziehen. Eine elegantere Herangehensweise zur Überprüfung der Forschungsfrage (effizientere und effektivere Verwaltung durch Verselbstständigung?) könnte darin bestehen, die Annahme zu treffen, dass die Management-Autonomie verselbstständigter Behörden einen positiven Einfluss auf deren administrative Leistungsfähigkeit hat. Hierdurch könnte auch vermieden werden, dass unterschiedliche Dimensionen organisatorischer Autonomie auf problematische Weise miteinander vermischt werden.

Die künstliche Unterscheidung von abhängigen Bundesoberbehörden bzw. traditionellen Verwaltungseinheiten und unabhängigen Bundesoberbehörden bzw. Agenturen führt letztlich dazu, dass die Überprüfung der grundlegenden These der Autoren, wonach organisatorische Verselbstständigung per se die funktionale Politisierung des Führungspersonals beeinflusst (S. 72, 75, 87), in der empirischen Analyse in den Hintergrund tritt. Diesen Effekt weisen Egeberg und Trondal (2009) anhand einer breit angelegten Befragung der norwegischen Zentralverwaltung nach. Sie zeigen, dass Beschäftigte in verselbstständigten Behörden politischen Signalen deutlich weniger Gewicht beimessen als Beschäftigte in der Ministerialverwaltung. Dieser Effekt ist unabhängig von der politischen Relevanz des Aufgabenbereichs und der eigenen hierarchischen Position nachweisbar. Egeberg und Trondal (2009) kommen ebenso wie Ebinger und Schmitt (S. 87-88) zu dem Ergebnis, dass das Ausmaß der öffentlichen Aufmerksamkeit von allen untersuchten Variablen den größten Einfluss auf die Beachtung politischer Signale der Beschäftigten in verselbstständigten Behörden besitzt. Möglicherweise wäre dieser Effekt in der Untersuchung von Ebinger und Schmitt noch deutlicher ausgefallen, wenn die Fallauswahl nicht anhand der politischen Relevanz der Behörden vorgenommen worden wäre. 
Die Ergebnisse zur Bedeutung der wahrgenommenen politischen Relevanz der eigenen Aufgabe für die funktionale Politisierung administrativer Führungskräfte machen zudem deutlich, dass verselbstständigte Behörden nicht nach einer betriebswirtschaftlichen Eigenlogik ,jenseits politischer Prärogativen operativ“ geführt werden (S. 75). Es wurde bereits darauf hingewiesen, dass eine derartige Abkoppelung der Verwaltung von der Politik kein Bestandteil des managementorientierten Modells verselbstständigter Behörden ist, sondern dieses ganz im Gegenteil auf eine größere politische Responsivität abzielt. Insofern stimmen die empirischen Ergebnisse durchaus positiv, denn gerade dann, wenn politische Ausstrahlungseffekte erwartbar sind, werden diese von den Führungskräften auch berücksichtigt.

\section{Schlussfolgerungen}

Ziel dieses Beitrags war es, zentrale konzeptionelle und theoretische Annahmen des Aufsatzes von Ebinger und Schmitt kritisch zu hinterfragen. Erstens konnte gezeigt werden, dass in der wissenschaftlichen Auseinandersetzung um verselbstständigte Behörden weitaus weniger begriffliche Verwirrung vorherrscht als von den Autoren angenommen. Insofern diente dieser Beitrag auch dazu, die beiden dominierenden Debatten zu verselbstständigten Behörden schärfer zu konturieren. Anschließend wurde herausgearbeitet, dass ein „Agenturfieber“ im bundesdeutschen Kontext kaum erkennbar ist. Ebenso wurde argumentiert, dass sich entgegen der Annahme der Autoren die Rechtsform verselbstständigter Behörden durchaus auf deren tatsächliche Autonomie auswirken kann. Zudem wurde gezeigt, dass die Ergebnisse der empirischen Analyse vorsichtig interpretiert werden sollten, da sowohl die Fallauswahl als auch die Unterscheidung zwischen traditionellen und modernen Agenturen als problematisch einzuschätzen sind. Schließlich wurde infrage gestellt, ob mithilfe des theoretischen Modells der Effekt organisatorischer Verselbstständigung auf administrative Leistungsfähigkeit untersucht werden kann.

In welchem Maße administrative Führungskräfte verselbstständigter Behörden politische Entscheidungskriterien antizipieren und bei der Wahrnehmung ihrer Aufgaben berücksichtigen ist eine spannende und bislang empirisch kaum untersuchte Fragestellung. Indem Ebinger und Schmitt sich ausschließlich auf die Literatur zu verselbstständigten Behörden beziehen, verkennen sie das Potenzial ihrer Daten. Dieses Potenzial liegt weniger darin, die Effekte managementorientierter Verwaltungsreformen in einem institutionellen Kontext nachzuweisen, in dem diese Reformen ohnehin keine besonders große Rolle spielen, sondern in der Auseinandersetzung mit den Rollenbildern, Einstellungen und Handlungsorientierungen administrativer Eliten in verselbstständigten Behörden. Im Unterschied zu den meisten empirischen Arbeiten zu verselbstständigten Behörden verfügen die Autoren über Daten zu Führungskräften aus verselbstständigten Behörden und Ministerialverwaltung; insofern drängt sich eine Analyse von Unterschieden und Gemeinsamkeiten zwischen administrativen Führungskräften in der Ministerialverwaltung und verselbstständigten Behörden unmittelbar auf. 


\section{Literatur}

Bach, Tobias. 2010. Policy and Management Autonomy of Federal Agencies in Germany. In Governance of Public Sector Organizations. Proliferation, Autonomy and Performance, Hrsg. Per Lægreid und Koen Verhoest, 89-110. Hampshire: Palgrave Macmillan.

Bach, Tobias, Julia Fleischer und Thurid Hustedt. 2010. Organisation und Steuerung zentralstaatlicher Behörden: Agenturen im westeuropäischen Vergleich. Berlin: Edition Sigma.

Bach, Tobias, und Etienne Huber 2012. Comparing Agencification in Continental Countries. In Government Agencies: Practices and Lessons from 30 Countries, Hrsg. Koen Verhoest, Sandra van Thiel, Geert Bouckaert und Per Lægreid, 203-210. Basingstoke: Palgrave Macmillan.

Bach, Tobias, und Werner Jann. 2010. Animals in the Administrative Zoo: Organizational Change and Agency Autonomy in Germany. International Review of Administrative Sciences 76:443-468.

Bouckaert, Geert, und B. Guy Peters. 2004. What is Available and what is Missing in the Study of Quangos? In Unbundled Government. A Critical Analysis of the Global Trend to Agencies, Quangos and Contractualisation, Hrsg. Christopher Pollitt und Colin Talbot, 22-49. London/New York: Routledge.

BT-Drs. 15/5111. 2005. Moderne Managementmethoden für eine moderne Verwaltung. Antwort der Bundesregierung vom 16.03.2005 auf die Große Anfrage der Abgeordneten Dr. Volker Wissing, Otto Fricke, Jürgen Koppelin, weiterer Abgeordneter und der Fraktion der FDP.

BT-Drs. 14/7033. 2001. Entwurf eines Gesetzes über die integrierte Finanzdienstleistungsaufsicht.

Christensen, Jørgen Grønnegård. 2001. Bureaucratic Autonomy as a Political Asset. In Politicians, Bureaucrats, and Administrative Reform, Hrsg. B. Guy Peters und Jon Pierre, 119-131. London/New York: Routledge.

Christensen, Tom, und Per Lægreid. 2006. Agencification and Regulatory Reforms. In Autonomy and Regulation: Coping with Agencies in the Modern State, Hrsg. Tom Christensen und Per Lægreid, 8-49. Cheltenham: Edward Elgar.

Christensen, Tom, und Per Lægreid. 2001. New Public Management. The Effects of Contractualism and Devolution on Political Control. Public Management Review 3:73-94.

Döhler, Marian. 2007a. Die politische Steuerung der Verwaltung. Baden-Baden: Nomos.

Döhler, Marian. 2007b. Vom Amt zur Agentur? Organisationsvielfalt, Anpassungsdruck und institutionelle Wandlungsprozesse im deutschen Verwaltungsmodell. In Agencies in Westeuropa, Hrsg. Werner Jann und Marian Döhler, 12-47. Wiesbaden: VS Verlag für Sozialwissenschaften.

Döhler, Marian. 2006. Regulative Politik und die Transformation der klassischen Verwaltung. In Politik und Verwaltung, PVS-Sonderheft 37, Hrsg. Jörg Bogumil, Werner Jann und Frank Nullmeier, 208-227. Wiesbaden: VS Verlag für Sozialwissenschaften.

Döhler, Marian. 2005. Die begrenzte Rationalität von Delegation und Steuerung in der Bundesverwaltung. In Mechanismen der Politik. Strategische Interaktionen im deutschen Regierungssystem, Hrsg. Steffen Ganghof und Philip Manow, 215-243. Frankfurt a. M./New York: Campus Verlag. 
Ebinger, Falk, und Carina Schmitt. 2010. Alles eine Frage des Managements? Wie Autonomierechte die Handlungsfreiheit des administrativen Führungspersonals beeinflussen. Politische Vierteljahresschrift 51:69-93.

Egeberg, Morten, und Jarle Trondal. 2009. Political Leadership and Bureaucratic Autonomy: Effects of Agencification. Governance 22:673-688.

Elgie, Robert. 2006. Why do Governments Delegate Authority to Quasi-autonomous Agencies? The Case of Independent Administrative Authorities in France. Governance 19:207-227.

Gilardi, Fabrizio. 2002. Policy Credibility and Delegation to Independent Regulatory Agencies: A Comparative Empirical Analysis. Journal of European Public Policy 9:873-893.

Gilardi, Fabrizio, und Dietmar Braun. 2002. Delegation aus der Sicht der Prinzipal-AgentTheorie. Politische Vierteljahresschrift 43:147-161.

Greve, Carsten, Matthew Flinders und Sandra Van Thiel. 1999. Quangos - What's in a name? Defining Quangos from a Comparative Perspective. Governance 12:129-146.

Hert, Katharina. 2009. Karrieremuster von Behördenleitern am Beispiel der Bundesoberbehörden. Potsdam: Universität Potsdam (unveröffentlichte BA-Arbeit).

Hood, Christopher. 1978. Keeping the Centre Small: Explanations of Agency Type. Political Studies 26:30-46.

Majone, Giandomenico. 1997. From the Positive to the Regulatory State: Causes and Consequences of Changes in the Mode of Governance. Journal of Public Policy 17:139-167.

Pollack, Mark A. 2002. Learning from the Americanists (Again): Theory and Method in the Study of Delegation. West European Politics 25:200-219.

Pollitt, Christopher, Karen Bathgate, Janice Caulfield, Amanda Smullen und Colin Talbot. 2001. Agency Fever? Analysis of an International Policy Fashion. Journal of Comparative Policy Analysis 3:271-290.

Pollitt, Christopher, Colin Talbot, Janice Caulfield und Amanda Smullen. 2004. Agencies: How Governments do Things Through Semi-Autonomous Organizations. London: Palgrave Macmillan.

Smullen, Amanda. 2004. Lost in Translation? Shifting Interpretations of the Concept of 'Agency': the Dutch Case. In Unbundled Government. A Critical Analysis of the Global Trend to Agencies, Quangos and Contractualisation, Hrsg. Christopher Pollitt und Colin Talbot, 184-202. London/New York: Routledge.

Thatcher, Mark. 2002. Delegation to Independent Regulatory Agencies: Pressures, Functions and Contextual Mediation. West European Politics 25:125-147.

Thynne, Ian. 2004. State Organisations as Agencies: an Identifiable and Meaningful Focus of Research? Public Administration and Development 24:91-99.

Verhoest, Koen, B. Guy Peters, Geert Bouckaert und Bram Verschuere. 2004. The Study of Organisational Autonomy: A Conceptual Review. Public Administration and Development 24:101-118.

Verhoest, Koen, Paul G. Roness, Bram Verschuere, Kristin Rubecksen und Muiris MacCarthaig. 2010. Autonomy and Control of State Agencies: Comparing States and Agencies. Hampshire: Palgrave Macmillan.

Verschuere, Bram. 2009. The Role of Public Agencies in the Policy Making Process: Rhetoric versus Reality. Public Policy and Administration 24:23-46. 
Wettenhall, Roger. 2005. Agencies and Non-departmental Public Bodies. The Hard and Soft Lenses of Agencification Theory. Public Management Review 7:615-635.

Yesilkagit, Kutsal, und Jørgen G. Christensen. 2010. Institutional Design and Formal Autonomy: Political versus Historical and Cultural Explanations. Journal of Public Administration Research and Theory 20:23-52.

\section{Autorenangaben:}

Dipl.-Verw. Wiss. Tobias Bach

Leibniz Universität Hannover, Institut für Politische Wissenschaft, Schneiderberg 50, 30167 Hannover,

bach@ipw.uni-hannover.de 